\title{
Association of rs2954029 and rs6982502 Variants with Coronary Artery Disease by HRM Technique: A GWAS Replication Study in an Iranian Population
}

\author{
Zahereh Karimi ${ }^{1}$, Javad Daneshmoghadam ${ }^{1}$, Hamid Ghaedi ${ }^{2}$, \\ Ehsan Khalili ${ }^{3}$, Ghodratollah Panahi*3 ${ }^{*}$, Mehrnoosh Shanaki*1
}

\begin{abstract}
Background: Genome-wide association studies (GWAS) have been the primary tool for an unbiased study of the genetic background of coronary artery disease (CAD). They have identified a list of single-nucleotide polymorphisms (SNPs) associated with coronary artery disease (CAD). In this study, we aimed to replicate the association of rs2954029 and rs6982502, a GWAS identified SNP, to CAD in an Iranian population.

Methods: A sample of 285 subjects undergoing coronary angiography, including 134 CAD patients and 151 healthy. The genotype determination of rs2954029 and rs6982502 SNPs performed using the high-resolution melting analysis (HRM) technique.

Results: Our results revealed that the TT genotype of $r 2954029(\mathrm{p}=0.009)$ and $\mathrm{rs} 6982502(\mathrm{p}<0.001)$ were significantly higher in CAD patients compared with controls. Binary logistic regression showed that rs6982502 and rs2954029 increase the risk of CAD incidence $(2.470$ times, $\mathrm{p}=0.011,95 \% \mathrm{CI}=[1.219$ 4.751], and 2.174 times, $\mathrm{p}=0.033,95 \% \mathrm{CI}=$ [1.066-4.433] respectively). After adjusting for confounders, we found that rs6982502 and rs2954029 are significantly associated with CAD risk.

Conclusions: These data showed that the TT genotype of rs2954029 and rs6982502 is associated with the risk of CAD in a hospital-based sample of the Iranian population, which has replicated the result of recent GWAS studies.
\end{abstract}

Keywords: Coronary Artery Disease (CAD), Genome-Wide Association Studies (GWAS), HighResolution Melting (HRM), Single-Nucleotide Polymorphisms (SNP).

\section{Introduction}

Coronary Artery Disease (CAD) is considered as a prominent cause of premature death in the world $(1,2)$. CAD is a chronic inflammatory disease in which immune function cells produce pro-inflammatory cytokines. The disease's main feature is the accumulation of lipids in the arterial wall, which leads to arterial blockage due to the formation of foam cells (3).
Risk factors for atherosclerosis include age, obesity, diabetes, high blood pressure, smoking, lifestyle, high cholesterol, and Low-Density Lipoprotein Cholesterol (LDL-C) content, and low blood High-Density Lipoprotein Cholesterol (HDL-C) content $(4,5)$.

Moreover, oxidative stress plays a crucial role in the progression of atherosclerosis (6).

1: Department of Medical Laboratory Sciences, School of Allied Medical Sciences, Shahid Beheshti University of Medical Sciences, Tehran, Iran.

2: Department of Medical Genetics, Faculty of Medicine, Shahid Beheshti University of Medical Sciences, Tehran, Iran.

3: Department of Clinical Biochemistry, Faculty of Medicine, Tehran University of Medical Sciences, Tehran, Iran.

*Corresponding author: Ghodratollah Panahi; Tel: +98 212272021; E-mail: a ghpanahi@sina.tums.ac.ir

\& Mehrnoosh Shanaki; Tel: +98 212272021; E-mail: shanaki_m@sbmu.ac.ir.

Received: 1 Mar, 2021; Accepted: 8 May, 2021 
Collected evidence suggests that the complex interactions between environmental risk factors and genes susceptible to CAD have a substantial role in CAD's pathogenesis. Most importantly, we cannot exclude the potential role of Single Nucleotide Polymorphism (SNP) in CAD's pathogenesis as the most common genetic variation among populations (7-9).

Genome-wide association studies (GWAS) have identified innumerable candidate genetic loci associated with the risk of CAD (10-13). In 2018, a study conducted a 1000 genome-based GWAS in which they identified 64 novel genetic risk loci for CAD (14). According to GWAS, TRIB1 is a CAD-associated locus (11, 15, 16). TRIB1, which is located on chromosome 8q24, encodes the protein tribbles homolog one that is recognized as an intriguing protein that is involved in the etiology of multiple human diseases; including myeloid leukemia, Crohn's disease, non-alcoholic fatty liver disease (NAFLD), dyslipidemia, and coronary artery disease (CAD) (17).

Based on GWAS, rs2954029 and rs6982502 are two intron variants near TRIB1 locus and are associated with cardiometabolic parameters, CAD, and other metabolic diseases (11). There are several studies on the relationship between CAD and polymorphisms in different populations. Still, to the best of our knowledge, few GWAS studies evaluated the association of these two SNPs with CAD in the Iranian population. Therefore, in this study, we investigate the correlation between TRIB1 genomic region SNPs, rs2954029 and rs6982502, with CAD susceptibility in the Iranian population as a replication study.

\section{Materials and Methods Study population}

This case-control study was performed on 134 Iranian patients with CAD and 151 healthy individuals whom all underwent angiography in Tehran Heart Center (Tehran University of Medical Sciences), Tehran, Iran. The ethics committee of Shahid Beheshti University of Medical Sciences approved the study protocols and methods
(IR.SBMU.RETECH.REC.1397.1177). The samples were collected between 2019 and 2020. Also, the study was following the declaration of Helsinki, and all patients signed written consent. The definitive diagnosis of CAD was made by a cardiologist using angiography. These patients were diagnosed with three-vessel CAD and candidates for coronary artery bypass graft (CABG) based on angiographic results. The severity of $\mathrm{CAD}$ was defined by $\geq 50 \%$ stenosis in at least one of the major coronary arteries. All participants were matched for age $(50 \pm 10)$. We selected non-CAD subjects from individuals with no coronary artery obstruction (zero) or a maximum of $5-10 \%$ stenosis in their coronary arteries referred to the mentioned clinic. We recruited all control subjects after careful examination by an angiography. All subjects were asked questions about their smoking habits, history of hypertension and diabetes, and complete medical history was obtained from their clinical records. We did not include the individuals with diabetes Mellitus and people with chronic diseases such as liver, kidney, stroke, myocardial infarction, open-heart surgery, and unstable angina. People who received vitamin $\mathrm{D}$ and antioxidant supplements in the previous 12 months and smokers were also not included.

\section{Measurements of biochemical parameters}

We measured systolic and diastolic blood pressure twice from each person's right arm. Subjects were instructed to rest for at least 10 minutes in a comfortable place; then, measurements were made (Omron, M6 Comfort HEM7321-E, Japan). Fasting blood sugar (FBS) was taken from CAD patients before coronary artery bypass graft (CABG). The assessment of fasting blood samples in the healthy control group was also carried out following the samples' collection after angiography. Total Cholesterol (TC), LDL-C, HDL-C, Triglyceride (TG), and FBS were quantified by routine laboratory kits (Pars Azmon Inc., Iran) in an auto-analyzer (Hitachi 917 Ltd, Tokyo, Japan). 


\section{DNA extraction}

DNA was separated by the salting-out method from whole blood $(18,19)$. After collecting DNA, the DNA concentration was specified by Nano Drop ${ }^{\mathrm{TM}}$ then the samples were stored at $-20{ }^{\circ} \mathrm{C}$ until utilization.

\section{Real-time PCR-HRM}

Real-time Polymerase Chain Reaction HighResolution Melting (real-time PCR-HRM) analysis was utilized to detect the TRIB1 LOC105375746 - rs2954029 polymorphism using forward: 5'- GCC ATT TAC AAA GCT GCT GAT GGT -3' and reverse: 5'-TTG TGT CAT GAG GGG AGA GAT ACA A -3' primers and to detect the TRIB1 LOC105375746 - rs6982502 polymorphism using forward: 5'- GAC GAC AGT TCC CTC CTT GG -3' and reverse: 5'-TGT CCT AGA TGT CCT TCA GCT C $-3^{\prime}$ primers. Primers for HRM were designed by Primer3web software (version 4.1.0.), and their specificity for PCR was checked by nucleotide BLAST (http://blast.ncbi.nlm.nih.gov/Blast.cgi).

To this end, the thermal cycling conditions were as follows: holding time, $95{ }^{\circ} \mathrm{C}$ for 12 min; followed by 35 denaturation cycles at 95 ${ }^{\circ} \mathrm{C}$ for $20 \mathrm{~s}$ and annealing for rs 2954029 at 58 ${ }^{\circ} \mathrm{C}$ and rs6982502 at $61{ }^{\circ} \mathrm{C}$ for $20 \mathrm{~s}$; extension at $72{ }^{\circ} \mathrm{C}$ for $20 \mathrm{~s}$; and HRM, 75-90 ${ }^{\circ} \mathrm{C}$. Then the HRM curves were normalized, and three different classes of melting curves were observed. Three samples with defined genotypes were subjected to Sanger sequencing to evaluate the reliability of the HRM method.

\section{Statistical Analysis}

We used the statistical software SPSS 26 (SPSS, Chicago, IL) for data analysis. Quantitative variables were tested for normality by Kolmogorov-Smirnov test. Quantitative variables were presented as median (min-max) deviation and tested by Mann-Whitney U test. Also, the $\chi 2$ test was employed to evaluate the deviation of genotype distribution from the HardyWeinberg equilibrium in both groups. We used binary logistic regression to assess the association of each SNP with the incidence of CAD. The association between CAD and the SNP genotypes and allelic frequencies was measured by the odds ratio (OR) with $95 \%$ confidence intervals (CI). The limit for statistical significance was maintained at $\mathrm{P}$ values $\leq 0.05$.

\section{Results}

\section{Demographics and clinical characteristics}

The clinical characteristics of CAD patients and the control group are shown in Table 1. The study groups were matched for age $(p=0.082)$. The subjects included 134 cases and 151 controls: $14.0 \%$ of the subjects were female, and $86.0 \%$ were male. Unfortunately, for the rs2954029, nine genotypes in the patient group and two genotypes in the control group could not be detected. The genotypes of these individuals were not mentioned in this article. Laboratory analysis showed that the serum level of LDL-C, WC, TC, and TG significantly increased in the patient group compared to the healthy subjects $(\mathrm{p}<0.001, \mathrm{p}=0.001, \mathrm{p}<0.001$, and $\mathrm{p}=0.001$ respectively). Also, systolic, and diastolic blood pressures were significantly higher in CAD patients than in the control group $(\mathrm{p}=0.035, \mathrm{p}=0.003$, respectively). Also, the healthy group showed significantly higher HDL-C ( $<<0.001$ and $p=0.001$, respectively) compared to CAD patients.

\section{Genotype and Allele Frequencies of rs2954029 and rs6982502}

Tables 2 and 3 lists the frequency of alleles and Hardy Weinberg equilibrium for variants rs2954029 and rs6982502, respectively. According to these tables, the genotype frequency of $\mathrm{rs} 2954029 \quad(\mathrm{p}=0.168)$ and rs6982502 ( $\mathrm{p}=0.126)$ agreed with the HardyWeinberg equilibrium. In addition, the $\mathrm{T}$ allele of rs2954029 and rs6982502 was higher in CAD compared to control group (OR 1.537; $95 \% \mathrm{CI}=[1.088-2.170], \mathrm{p}=0.015$ and $\mathrm{OR}$ $1.904 ; 95 \% \mathrm{CI}=[1.360-2.667], \mathrm{p}=0.001$ respectively). Based on our analysis, the TT genotype frequency was significantly higher in CAD patients compared with controls in both rs2954029 and rs6982502 variants $(\mathrm{p}=0.009$, 
$\mathrm{p}<0.001)$. Table 4 analyzed the association of rs2954029 and rs6982502 genotype with CAD and presented three genetic models (Dominant, Recessive, and Over-dominant) for these SNPs. Moreover, our results showed that the TT+AT genotypes in rs2954029 and the $\mathrm{TT}+\mathrm{CT}$ in rs6982502 were more frequent in CAD patients compared to controls under the dominant model (OR 0.60; 95\% CI= [0.361- 0.996], $\mathrm{p}=0.047$ and OR 2.286; 95\% $\mathrm{CI}=[1.358-3.848], \mathrm{p}=0.002)($ Table 4). In table 5, binary logistic regression results showed that rs6982502, 2.470 times $(\mathrm{p}=0.011$, 95\% $\mathrm{CI}=[1.219-4.751])$ and $\mathrm{rs} 2954029,2.174$ times $(\mathrm{p}=0.033,95 \% \quad \mathrm{CI}=[1.066-4.433])$ increases the risk of CAD incidence.

Table 1. Demographic and clinical characteristics of the study population.

\begin{tabular}{lllc}
\hline Parameter & $\begin{array}{l}\text { CAD patients } \\
\text { (n= 134) } \\
\text { Median (min-max) }\end{array}$ & $\begin{array}{l}\text { Controls } \\
\text { (n= 151) } \\
\text { Median (min-max) }\end{array}$ & $\begin{array}{c}\text { p-value } \\
\text { Mann-Whitney test }\end{array}$ \\
\hline Sex (male/female) & $116 / 18$ & $129 / 22$ & 0.783 \\
Age (year) & $56(45-60)$ & $55(45-60)$ & 0.082 \\
FBS (mg/dL) & $92(68-173)$ & $93(63-194)$ & 0.926 \\
BMI $\left(\mathrm{kg} / \mathrm{m}^{2}\right)$ & $26.29(19.14-38.80)$ & $26.15(19-37.11)$ & 0.995 \\
WC $(\mathrm{cm})$ & $98.5(84-117)$ & $91.5(72-114)$ & $\mathbf{0 . 0 0 1}$ \\
LDL-C $(\mathrm{mg} / \mathrm{dL})$ & $102.67(63-233)$ & $78.05(51-102)$ & $<\mathbf{0 . 0 0 1}$ \\
HDL-C (mg/dL) & $35(19-89)$ & $40(17-480)$ & $<\mathbf{0 . 0 0 1}$ \\
TC $(\mathrm{mg} / \mathrm{dL})$ & $163.5(103-264)$ & $137(76-282)$ & $<\mathbf{0 . 0 0 1}$ \\
TG $(\mathrm{mg} / \mathrm{dL})$ & $140(42-382)$ & $111(23-487)$ & $\mathbf{0 . 0 0 1}$ \\
Ca $(\mathrm{mg} / \mathrm{dL})$ & $9.3(7.8-10.4)$ & $9.6(9.1-10.2)$ & 0.051 \\
Phos $(\mathrm{mg} / \mathrm{dL})$ & $3.03(1.48-5.70)$ & $3.26(2.03-5.50)$ & 0.057 \\
Vit D (ng/mL) & $26.36(3.76-140.3)$ & $36.39(7.15-120.68)$ & 0.007 \\
SBP $(\mathrm{mmHg})$ & $120(100-170)$ & $120(110-130)$ & $\mathbf{0 . 0 3 5}$ \\
DBP $(\mathrm{mmHg})$ & $80(60-100)$ & $75(60-80)$ & $\mathbf{0 . 0 0 3}$ \\
\hline
\end{tabular}

CAD: coronary artery disease, FBS: fast blood sugar, BMI: body mass index, WC: waist circumference, LDL-C: lowdensity lipoprotein cholesterol, HDL-C: high-density lipoprotein cholesterol, TC: total cholesterol, TG: triglycerides, Ca: calcium, Phos: phosphorous, Vit D: vitamin D, SBP: systolic blood pressure, DBP: diastolic blood pressure. Data are shown as median $\pm \mathrm{IQR}$ (interquartile range).

Table 2. Genotype distribution and relative allele frequencies of the rs2954029 A>T variants.

\begin{tabular}{lllll}
\hline \multirow{2}{*}{ Genotypes } & $\begin{array}{l}\text { Control } \\
\mathbf{N = 1 4 9}\end{array}$ & $\begin{array}{l}\text { CAD } \\
\mathbf{N = 1 2 5}\end{array}$ & p-value* & OR (95\% CI) \\
\cline { 2 - 5 } & $\mathbf{N}(\mathbf{\%})$ & $\mathbf{N}(\mathbf{\%})$ & & \\
\hline AA & $60(40.30 \%)$ & $36(28.80 \%)$ & $\mathbf{0 . 0 3 5}$ & - \\
\hline AT & $75(50.30 \%)$ & $66(52.80 \%)$ & 0.153 & $0.682(0.402-1.158)$ \\
\hline TT & $14(9.40 \%)$ & $23(18.40 \%)$ & $\mathbf{0 . 0 0 9}$ & $2.738(1.252-5.987)$ \\
\hline Allele & & & & \\
\hline A & $195(65.4 \%)$ & $138(55.2 \%)$ & & \\
\hline T & $103(34.6 \%)$ & $112(44.8 \%)$ & $\mathbf{0 . 0 1 5}$ & $1.537(1.088-2.170)$ \\
\hline
\end{tabular}

*p values and OR were computed by $\chi 2$ tests. OR: odds ratio, CI: confidence interval. 
Table 3. Genotype distribution and relative allele frequencies of the rs6982502 C>T variants.

\begin{tabular}{lllll}
\hline \multirow{2}{*}{ Genotypes } & $\begin{array}{l}\text { Control } \\
\text { N= 151 }\end{array}$ & $\begin{array}{l}\text { CAD } \\
\text { N= 134 }\end{array}$ & \multirow{2}{*}{ p value * } & OR (95\% CI) \\
\cline { 2 - 4 } & $\mathbf{N}(\mathbf{\%})$ & $\mathbf{N}(\mathbf{\%})$ & & \\
\hline CC & $60(39.70 \%)$ & $30(22.40 \%)$ & $\mathbf{0 . 0 0 0}$ & - \\
\hline CT & $77(51.0 \%)$ & $73(54.50 \%)$ & $\mathbf{0 . 0 2 0}$ & $1.896(1.102-3.263)$ \\
\hline TT & $14(9.30 \%)$ & $31(23.10 \%)$ & $\mathbf{0 . 0 0 0}$ & $4.429(2.054-9.549)$ \\
\hline Allele & & & & \\
\hline C & $197(65.2 \%)$ & $133(49.6 \%)$ & & $1.904(1.360-2.667)$ \\
\hline T & $105(34.8 \%)$ & $135(50.4 \%)$ & $\mathbf{0 . 0 0 0}$ & \\
\hline HWE & & & 0.126 & \\
\hline
\end{tabular}

*p values and OR were computed by $\chi 2$ tests. OR: odds ratio, CI: confidence interval.

Table 4. Dominant, recessive, and Over-dominant models in case-control studies.

\begin{tabular}{|c|c|c|c|c|c|c|}
\hline & Genotypes & Models & $\begin{array}{l}\text { Control } \\
\mathbf{N}(\%)\end{array}$ & $\begin{array}{l}\text { CAD } \\
\mathbf{N}(\%)\end{array}$ & OR $(95 \% \mathrm{CI})$ & $\begin{array}{c}\text { p- } \\
\text { value* }\end{array}$ \\
\hline \multirow{6}{*}{ Rs2954029 } & $\mathrm{TT}+\mathrm{AT}$ & \multirow{2}{*}{ Dominant } & $89(59.70 \%)$ & $99(71.20 \%)$ & \multirow{2}{*}{$0.60(0.361-0.996)$} & \multirow{2}{*}{0.047} \\
\hline & AA & & $60(40.30 \%)$ & $36(28.80 \%)$ & & \\
\hline & TT & \multirow{2}{*}{ Recessive } & $14(9.40 \%)$ & $23(18.40 \%)$ & \multirow{2}{*}{$2.174(1.066-4.433)$} & \multirow{2}{*}{0.030} \\
\hline & $\mathrm{AA}+\mathrm{AT}$ & & $135(90.60 \%)$ & $102(81.60 \%)$ & & \\
\hline & AT & \multirow{2}{*}{ Over-dominant } & $75(50.30 \%)$ & $66(52.80 \%)$ & \multirow{2}{*}{$0.906(0.563-1.458)$} & \multirow{2}{*}{0.684} \\
\hline & $\mathrm{AA}+\mathrm{TT}$ & & $74(49.70 \%)$ & $59(47.20 \%)$ & & \\
\hline \multirow{6}{*}{ Rs6982502 } & $\mathrm{TT}+\mathrm{CT}$ & \multirow{2}{*}{ Dominant } & $91(60.30 \%)$ & $104(77.60 \%)$ & \multirow{2}{*}{$2.286(1.358-3.848)$} & \multirow{2}{*}{0.002} \\
\hline & $\mathrm{CC}$ & & $60(39.70 \%)$ & $30(22.40 \%)$ & & \\
\hline & TT & \multirow{2}{*}{ Recessive } & $14(9.30 \%)$ & $31(23.10 \%)$ & \multirow{2}{*}{$2.945(1.491-5.819)$} & \multirow{2}{*}{0.001} \\
\hline & $\mathrm{CC}+\mathrm{CT}$ & & $137(90.70 \%)$ & $103(76.90 \%)$ & & \\
\hline & CT & \multirow{2}{*}{ Over-dominant } & $77(51.0 \%)$ & $73(54.50 \%)$ & \multirow{2}{*}{$0.869(0.545-1.386)$} & \multirow{2}{*}{0.557} \\
\hline & $\mathrm{TT}+\mathrm{CC}$ & & $74(49.00 \%)$ & $61(45.50 \%)$ & & \\
\hline
\end{tabular}

*p values and OR were computed by $\chi 2$ tests. OR: odds ratio, CI: confidence interval.

Table 5. Logistic regression analysis of the rs2954029 and rs6982502 variants with the risk of CAD.

\begin{tabular}{lllll}
\cline { 2 - 4 } & \multicolumn{1}{c}{ p value } & OR & 95\% CI \\
\hline SNP rs6982502 & 0.011 & 2.470 & $1.219-4.751$ \\
SNP rs2954029 & 0.033 & 2.174 & $1.066-4.433$ \\
\hline
\end{tabular}

CI: confidence interval, OR: odds ratio.

\section{Discussion}

Coronary artery disease (CAD) is, in fact, one of the most common causes of mortality worldwide. Besides lifestyle and environmental factors, which play an essential role in CAD development (20-22), a genetic basis has been established for CAD (23). GWAS studies have detected strong associations among different loci and CAD (24). Over the past decade, GWAS has shown that chromosomal locations play a vital role in increasing CAD sensitivity (24). In the present study, we surveyed the Iranian population to evaluate CAD's association with TRIB1 rs2954029 and rs6982502 variants adjacent to the TRIB1 risk locus derived from GWAS based on 1000 genomes.

Most importantly, we observed that that the genotypes of rs2954029 and rs6982502 were significantly associated with the risk of CAD. T allele and TT genotype of the two variants were significantly more frequent in CAD patients than healthy controls. Moreover, the TT+AT genotypes of rs2954029 and the TT+CT of rs6982502 were 
more frequent in CAD patients than healthy controls under the dominant model.

These results were partly consistent with previous studies in the other populations. Ram et al. identified that rs6982502 is associated with hypertriglyceridemia in ethnic Arabs, a significant risk factor for CAD (25).

Regarding the observed higher frequency of rs6982502 $\mathrm{T}$ allele in our CAD patients, in a study conducted by Ishizuka et al. in the Japanese population, they concluded that the $\mathrm{C}$ minor allele rs6982502 increased the risk of non-alcoholic fatty liver disease (NAFLD), which is another metabolic disorder which has some shared pathophysiological mechanisms with CAD (26).

Although Varbo et al. and Zhang et al. showed in two different studies that TRIB1rs2954029 was linked with the increased risk of ischemic heart disease in the general population and Chinese people, respectively $(27,28)$, in contrast to our results indicating a high frequency of TT genotype in CAD patients; Varbo et al. showed that TRIB1rs2954029 TA genotype was associated with an increased level of TG and ischemic heart disease (27). Moreover, Zhang et al. showed that A allele of TRIB1-rs2954029 was associated with blood lipids and risk of ischemic heart disease (28). Contrary to our findings, Toshiyuki Ikeoka et al. showed that the AA genotype of TRIB1 rs2954029 was associated with TG serum level in Japanese women (29). Population differences in genotype frequencies of SNPs in TRIB1 might explain this discrepancy.

Like other complex disorders, many CADassociated risk variants have been discovered by several GWAS (30). Based on GWAS results, in a sample of coronary artery disease cases, Willer et al. identified that the $\mathrm{T}$ allele of TRIB1 rs2954029 is associated with lipid concentrations and risk of CAD (31). The GWAS results by Teslovich et al. in European ancestry showed that the TRIB1 rs2954029 T allele affects blood lipids and has increased the risk of $\mathrm{CAD}$, which is consistent with our results (32).
Moreover, Different GWAS results showed that TRIB1 rs2954029 affects the concentration of TC, TG, LDL-C, and HDL$\mathrm{C}$ in different populations $(28,32,33)$. These findings favor our results about the higher TC and LDL-C in CAD patients than in the control group.

TRIB1, located on chromosome 8 (8q24.13), encodes a G-protein-coupled receptor-induced protein that plays a role in the mitogen-activated protein kinases(MAPK-) related signaling cascade, which mediates cell proliferation, differentiation, and apoptosis, and can regulate lipid metabolism through this pathway $(34,35)$. It is also known to be a risk factor for CAD (36). TRIB1 is associated with changes in serum levels of various factors such as TC, LDL-C, HDL-C, TG, and CAD (37). TRIB1 regulates smooth muscle cell proliferation and vascular chemotaxis via the Jun kinase pathway. Its overexpression in human atherosclerotic arteries with chronic inflammation suggests that TRIB1 may play an essential role in CAD and atherosclerosis (38). Recent studies have shown that several SNPs in TRIB1 were associated with one or more lipid parameters and CAD (39).

One possible explanation for the association of TRIB1 locus with lipid metabolism may be the regulatory effects of TRIB1 on mitogen-activated protein kinase that leads to dyslipidemia, but the exact mechanism is still unknown. It has also been postulated that TRIB1 controls chemotaxis and proliferation of smooth muscle cells in the arterial intima, and through this, it may lead to lipoprotein-independent atherosclerosis $(27,28)$. Rest upon the evidence from the previous human and animal studies and the results of our study; one may conclude that TRIB1 and near SNPs are involved in lipid metabolism and atherosclerosis $(14,27,32)$.

Studies with a larger sample size and based on various ethnic groups might be more reproducible. Because lifestyle information, such as eating habits and physical activities, 
could not be fully collected, potential geneenvironmental interactions could not be eliminated. Lifestyle modification with the guidance of a health care team led by a physician may have a significant positive impact on the future of cardiovascular and vascular events.

For the first time, we found that both TRIB1 adjacent variants rs2954029 and rs6982502 were significantly associated with lipid profile and increased risk of coronary artery disease in Iranian population. Further

\section{References}

1. Rashid MA, Edwards D, Walter FM, Mant J. Medication taking in coronary artery disease: a systematic review and qualitative synthesis. Ann Fam Med. 2014;12(3):224-32.

2. Shanaki M, Hossein-Nezhad A, Meshkani R, Beigy M, Shirzad M, Pasalar P, et al. Effects of resveratrol on crosstalk between canonical Bcatenin/Wnt and FOXO pathways in coronary artery disease patients with metabolic syndrome: a case control study. Iran $\mathbf{J}$ Pharm Res. 2016;15(3):547-559.

3. Rafieian-Kopaei M, Setorki M, Doudi M, Baradaran A, Nasri H. Atherosclerosis: process, indicators, risk factors and new hopes. Int J Prev Med. 2014;5(8):927-46.

4. Ebrahimi M, Kazemi-Bajestani S, GhayourMobarhan M, Ferns G. Coronary artery disease and its risk factors status in Iran: a review. Iran Red Crescent Med J. 2011;13(9):610-23.

5. Kazemi T, Sharifzadeh G, Javadinia SA, Salehiniya H. Prevalence of cardiovascular risk factors among the nurse population in the east of Iran. International Journal of Travel Medicine and Global Health. 2015;3(4):133-136.

6. Yang X, Li Y, Li Y, Ren X, Zhang X, Hu D, et al. Oxidative stress-mediated atherosclerosis: mechanisms and therapies. Front Physiol. 2017;8:600.

7. Bampali K, Mouzarou A, Lamnisou K, Babalis D. Genetics and coronary artery disease: present and future. Hellenic $\mathrm{J}$ Cardiol. 2014;55(2):156-63.

8. Cen J, Xiong Q, Yang X, Guo J, Xiong X-d, Zhang A. Pri-miR-34b/c rs4938723 Polymorphism Contributes to Coronary Artery studies in more extensive and multiple ethnic populations are needed to confirm the present data.

\section{Acknowledgements}

We are thankful to all participants of this study for their contribution to our study. This study was generously supported by Shahid Beheshti University of Medical Sciences Grant for MSc thesis (ID: 17011). The authors declare no conflict of interest.

Disease Susceptibility. Arch Med Res. 2019;50(4):170-174.

9. Peden JF, Farrall M. Thirty-five common variants for coronary artery disease: the fruits of much collaborative labour. Hum Mol Genet. 2011;20(R2):R198-205.

10. Deloukas P, Kanoni S, Willenborg C, Farrall M, Assimes TL, Thompson JR, et al. Large-scale association analysis identifies new risk loci for coronary artery disease. Nat Genet. 2011;43(4):333-338.

11. Jadhav KS, Bauer RC. Trouble With Tribbles-1: Elucidating the Mechanism of a Genome-Wide Association Study Locus. Arteriosclerosis, thrombosis, and vascular biology. 2019;39(6):998-1005.

12. Kessler T, Vilne B, Schunkert H. The impact of genome-wide association studies on the pathophysiology and therapy of cardiovascular disease. EMBO Mol Med. 2016;8(7):688-701.

13. Nikpay M, Goel A, Won H-H, Hall LM, Willenborg C, Kanoni S, et al. A comprehensive 1000 Genomes-based genome-wide association meta-analysis of coronary artery disease. Nat Genet. 2015;47(10):1121-1130.

14. van der Harst P, Verweij N. Identification of 64 novel genetic loci provides an expanded view on the genetic architecture of coronary artery disease. Circ Res. 2018;122(3):433-443.

15. Douvris A, Soubeyrand S, Naing T, Martinuk A, Nikpay M, Williams A, et al. Functional Analysis of the TRIB 1 Associated Locus Linked to Plasma Triglycerides and Coronary Artery Disease. J Am Heart Assoc. 2014;3(3):e000884. 
16. Nelson CP, Goel A, Butterworth AS, Kanoni $\mathrm{S}$, Webb TR, Marouli E, et al. Association analyses based on false discovery rate implicate new loci for coronary artery disease. Nat Genet. 2017;49(9):1385-1391.

17. Bauer RC, Yenilmez BO, Rader DJ. Tribbles1: a novel regulator of hepatic lipid metabolism in humans. Biochem Soc Trans. 2015;43(5):107984.

18. Nasiri H, Forouzandeh M, Rasaee M, Rahbarizadeh F. Modified salting-out method: high-yield, high-quality genomic DNA extraction from whole blood using laundry detergent. J Clin Lab Anal. 2005;19(6):229-32.

19. Rivero ER, Neves AC, Silva-Valenzuela MG, Sousa SO, Nunes FD. Simple salting-out method for DNA extraction from formalin-fixed, paraffin-embedded tissues. Pathol Res Pract. 2006;202(7):523-9.

20. Lopez AD, Mathers CD, Ezzati M, Jamison DT, Murray CJ. Global and regional burden of disease and risk factors, 2001: systematic analysis of population health data. Lancet. 2006;367(9524):1747-57.

21. Samani NJ, Erdmann J, Hall AS, Hengstenberg C, Mangino M, Mayer B, et al. Genomewide association analysis of coronary artery disease. N Engl J Med. 2007;357(5):44353.

22. Yusuf S, Hawken S, Ounpuu S, Dans T, Avezum A, Lanas F, et al. Investigators IS. Effect of potentially modifiable risk factors associated with myocardial infarction in 52 countries (the INTERHEART study): case-control study. Lancet. 2004;364(9438):937-52.

23. Munz M, Richter GM, Loos BG, Jepsen S, Divaris K, Offenbacher S, et al. Genome-wide association meta-analysis of coronary artery disease and periodontitis reveals a novel shared risk locus. Sci Rep. 2018;8(1):13678.

24. Jones PD, Webb TR. From GWAS to new biology and treatments in CAD. Aging (Albany NY). 2019;11(6):1611-1612.

25. Ram R, Wakil S, Muiya N, Andres E, Mazhar $\mathrm{N}$, Hagos $\mathrm{S}$, et al. A common variant association study in ethnic Saudi Arabs reveals novel susceptibility loci for hypertriglyceridemia. Clin Genet. 2017;91(3):371-378.
26. Ishizuka $Y$, Nakayama $K$, Ogawa $A$, Makishima S, Boonvisut S, Hirao A, et al. TRIB1 downregulates hepatic lipogenesis and glycogenesis via multiple molecular interactions. J Mol Endocrinol. 2014;52(2):145-58.

27. Varbo A, Benn M, Tybjærg-Hansen A, Grande P, Nordestgaard BG. TRIB1 and GCKR polymorphisms, lipid levels, and risk of ischemic heart disease in the general population. Arterioscler Thromb Vasc Biol. 2011;31(2):4517.

28. Zhang Z, Tao L, Chen Z, Zhou D, Kan M, Zhang D, et al. Association of genetic loci with blood lipids in the Chinese population. PLoS One. 2011;6(11):e27305.

29. Ikeoka T, Hayashida N, Nakazato M, Sekita $\mathrm{T}$, Murata-Mori F, Ando $\mathrm{T}$, et al. The A> T polymorphism of the tribbles homolog 1 gene is associated with serum triglyceride concentrations in Japanese community-dwelling women. Tohoku J Exp Med. 2014;233(2):149-53.

30. Cheema AN, Rosenthal SL, Kamboh IM. Proficiency of data interpretation: identification of signaling SNPs/specific loci for coronary artery disease. Database. 2017;2017.

31. Willer CJ, Sanna S, Jackson AU, Scuteri A, Bonnycastle LL, Clarke R, et al. Newly identified loci that influence lipid concentrations and risk of coronary artery disease. Nat Genet. 2008;40(2):161-9.

32. Teslovich TM, Musunuru K, Smith AV, Edmondson AC, Stylianou IM, Koseki M, et al. Biological, clinical and population relevance of 95 loci for blood lipids. Nature. 2010;466(7307):707-713.

33. Waterworth DM, Ricketts SL, Song K, Chen L, Zhao JH, Ripatti S, et al. Genetic variants influencing circulating lipid levels and risk of coronary artery disease. Arterioscler Thromb Vasc Biol. 2010;30(11):2264-76.

34. Hegedus Z, Czibula A, Kiss-Toth E. Tribbles: a family of kinase-like proteins with potent signalling regulatory function. Cell Signal. 2007;19(2):238-50.

35. Vilkeviciute A, Kriauciuniene L, Chaleckis R, Deltuva VP, Liutkeviciene R. RAD51B (rs8017304 and rs2588809), TRIB1 (rs6987702, rs4351379, and rs4351376), COL8A1 (rs13095226), and COL10A1 (rs1064583) gene 
variants with predisposition to age-related macular degeneration. Dis Markers. 2019;2019:5631083.

36. Iwamoto S, Boonvisut S, Makishima S, Ishizuka Y, Watanabe K, Nakayama K. The role of TRIB1 in lipid metabolism; from genetics to pathways. Biochem Soc Trans. 2015;43(5):10638.37. Consortium IKC. Large-scale genecentric analysis identifies novel variants for coronary artery disease. PLoS Genet. 2011;7(9):e1002260.
38. Sung HY, Guan H, Czibula A, King AR, Eder $\mathrm{K}$, Heath $\mathrm{E}$, et al. Human tribbles-1 controls proliferation and chemotaxis of smooth muscle cells via MAPK signaling pathways. J Biol Chem. 2007;282(25):18379-18387.

39. Sallam T, Sandhu J, Tontonoz P. Long noncoding RNA discovery in cardiovascular disease: decoding form to function. Circ Res. 2018;122(1):155-166. 